Tohoku Math. J.

68 (2016), 273-291

\title{
HOLOMORPHIC EQUIVALENCE PROBLEM FOR REINHARDT DOMAINS AND THE CONJUGACY OF TORUS ACTIONS
}

\author{
SATORU SHIMIZU
}

(Received November 15, 2011, revised November 4, 2014)

\begin{abstract}
In this paper, we give an answer to the holomorphic equivalence problem for a basic class of unbounded Reinhardt domains. As an application, we show the conjugacy of torus actions on such a class of Reinhardt domains, and discuss the relation between the holomorphic equivalence problem for Reinhardt domains and the conjugacy of torus actions.
\end{abstract}

Introduction. Torus actions are used in various field of mathematics. Meanwhile, torus actions themselves have very interesting properties. The conjugacy of torus actions (cf. Section 4) is one of such properties and has fundamental importance in the study of torus actions. When we are discussing torus actions on complex manifolds, the investigation of the conjugacy of torus actions has a close connection with some problem on several complex variables. In fact, the holomorphic equivalence problem for Reinhardt domains in several complex variables is nothing but the investigation of the conjugacy of torus actions on a Reinhardt domain. The purpose of this paper is to clarify this fact by giving an answer to the holomorphic equivalence problem for a basic class of unbounded Reinhardt domains. Our results in this paper, together with those in a sequent of papers [3], [4], [5], [6], [7], give illustrative examples of the phenomenon that, in complex analysis, when some object admits a torus action, its torus action direct an interplay between the analytic and algebraic structures of the object.

This paper is organized as follows. In Section 1, we recall basic concepts and results on Reinhardt domains, and state as Theorem 1.2 a main result of this paper on the holomorphic equivalence problem for a basic class of unbounded Reinhardt domains, which generalizes results of [5], [6]. Our proof of Theorem 1.2 is done by making use of tube domains which are covering manifolds of Reinhardt domains. In a sense, Reinhardt domains are rigid for doing a change of coordinates, while tube domains are rather flexible for that. We utilize this flexibility of tube domains. Observations on tube domains needed to prove Theorem 1.2 are given in Section 2 together with a lemma from linear algebra. Section 3 is devoted to the proof of Theorem 1.2. The conjugacy of torus actions on a Reinhardt domain is discussed in Section 4. Section 5 gives a remark on automorphisms of unbounded Reinhardt domains obtained from the proof of Theorem 1.2.

2010 Mathematics Subject Classification. Primary 32A07; Secondary 32M05.

Key words and phrases. Holomorphic equivalence problem, Reinhardt domains, Torus actions.

Partly supported by the Grant-in-Aid for Scientific Research (C), Japan Society for the Promotion of Science. 
1. Holomorphic equivalence problem for Reinhardt domains. We first collect some notations and terminology. As a general notational convention, we denote elements of $\mathbf{Z}^{n}, \mathbf{R}^{n}$, or $\mathbf{C}^{n}$ by column vectors. When dealing with matrices, we denote by $I_{p}$ and $O$ the unit matrix of degree $p$ and the zero matrix, respectively. The diagonal matrix of degree $n$ with the $i$-th diagonal entry $\alpha_{i}$ is denoted by diag $\left(\alpha_{1}, \ldots, \alpha_{n}\right)$. For an $n \times l$ matrix $M=\left(v_{i j}\right)$ with $n>l$ and an $l$-tuple $i_{1}, \ldots, i_{l}$ of indices with $1 \leq i_{1}<\cdots<i_{l} \leq n$, we define an $l \times l$ matrix $M\left(i_{1}, \ldots, i_{l}\right)$ by

$$
M\left(i_{1}, \ldots, i_{l}\right)=\left(\begin{array}{ccc}
v_{i_{1} 1} & \cdots & v_{i_{1} l} \\
\vdots & & \vdots \\
v_{i_{l} 1} & \cdots & v_{i_{l} l}
\end{array}\right) .
$$

The set of non-zero complex numbers is denoted by $\mathbf{C}^{*}$. The multiplicative group of complex numbers of absolute value 1 is denoted by $U(1)$. An automorphism of a complex manifold $M$ means a biholomorphic mapping of $M$ onto itself. The group of all automorphisms of $M$ is denoted by $\operatorname{Aut}(M)$. Two complex manifolds are said to be holomorphically equivalent if there is a biholomorphic mapping between them.

We now recall basic concepts and results on Reinhardt domains (cf. Shimizu [3], [4]). Write $T=(U(1))^{n}$. The group $T$ acts on $\mathbf{C}^{n}$ as a group of automorphisms by the standard rule, i.e.,

$$
\alpha \cdot z={ }^{t}\left(\alpha_{1} z_{1}, \ldots, \alpha_{n} z_{n}\right) \text { for } \alpha={ }^{t}\left(\alpha_{1}, \ldots, \alpha_{n}\right) \in T \text { and } z={ }^{t}\left(z_{1}, \ldots, z_{n}\right) \in \mathbf{C}^{n} .
$$

By definition, a Reinhardt domain $D$ in $\mathbf{C}^{n}$ is a domain in $\mathbf{C}^{n}$ which is stable under the action of $T$, that is, $\alpha \cdot D \subset D$ for all $\alpha \in T$. The group $T$ then acts on $D$ as a group of automorphisms. The subgroup of $\operatorname{Aut}(D)$ induced by the action of $T$ is denoted by $T(D)$.

An automorphism $\varphi$ of $\left(\mathbf{C}^{*}\right)^{n}$ is called an algebraic automorphism if the components of $\varphi$ are given by Laurent monomials, that is, $\varphi$ is of the form

$$
\begin{gathered}
\varphi:\left(\mathbf{C}^{*}\right)^{n} \ni^{t}\left(z_{1}, \ldots, z_{n}\right) \longmapsto{ }^{t}\left(w_{1}, \ldots, w_{n}\right) \in\left(\mathbf{C}^{*}\right)^{n}, \\
w_{i}=\alpha_{i} z_{1}^{a_{i 1}} \cdots z_{n}^{a_{i n}}, \quad i=1, \ldots, n,
\end{gathered}
$$

where $\left(a_{i j}\right) \in G L(n, \mathbf{Z})$ and $\left(\alpha_{i}\right) \in\left(\mathbf{C}^{*}\right)^{n}$. The set Aut alg $\left(\left(\mathbf{C}^{*}\right)^{n}\right)$ of all algebraic automorphisms of $\left(\mathbf{C}^{*}\right)^{n}$ forms a subgroup of $\operatorname{Aut}\left(\left(\mathbf{C}^{*}\right)^{n}\right)$.

Let $\varphi$ be an algebraic automorphisms of $\left(\mathbf{C}^{*}\right)^{n}$ and write $\varphi(z)={ }^{t}\left(\varphi_{1}(z), \ldots, \varphi_{n}(z)\right)$. In general, the components $\varphi_{1}, \ldots, \varphi_{n}$ have zeroes or poles along each coordinate hyperplane. If, for two domains $D$ and $D^{\prime}$ in $\mathbf{C}^{n}$ not necessarily contained in $\left(\mathbf{C}^{*}\right)^{n}$, they have no poles on $D$ and $\varphi: D \rightarrow \mathbf{C}^{n}$ maps $D$ biholomorphically onto $D^{\prime}$, then we say that $\varphi$ induces a biholomorphic mapping of $D$ onto $D^{\prime}$.

Consider a biholomorphic mapping $\varphi: D \rightarrow D^{\prime}$ between two Reinhardt domains $D$ onto $D^{\prime}$ in $\mathbf{C}^{n}$. The following proposition gives a necessary and sufficient condition for $\varphi$ to be equivariant with respect to the $T$-actions.

PROPOSITION 1.1 (cf. [4, Section 2]). The mapping $\varphi$ is induced by an algebraic automorphism of $\left(\mathbf{C}^{*}\right)^{n}$ if and only if $\varphi T(D) \varphi^{-1}=T\left(D^{\prime}\right)$. 
Biholomorphic mappings between Reinhardt domains equivariant with respect to the $T$ actions induce a natural equivalence relation between Reinhardt domains. In view of this obsevation, we say that two Reinhardt domains in $\mathbf{C}^{n}$ are algebraically equivalent if there is a biholomorphic mapping between them induced by an algebraic automorphism of $\left(\mathbf{C}^{*}\right)^{n}$. We are led to the following problem.

Problem $(*)$ (Holomorphic equivalence problem for Reinhardt domains). If two Reinhardt domains $D$ and $D^{\prime}$ in $\mathbf{C}^{n}$ are holomorphically equivalent, then are they algebraically equivalent?

The answer to this problem is affirmative when $D$ and $D^{\prime}$ are bounded [3, Section 4 , Theorem 1]. Furthermore, the proof in [3] is also valid for the class of Reinhardt domains whose automorphism groups have the structure of Lie groups with respect to the compact-open topology, which includes the class of Reinhardt domains that are holomorphically equivalent to bounded domains. In the proof, we used the conjugacy of the torus actions on such Reinhardt domains, which will be discussed in Section 4.

On the other hand, the unbounded case, that is, the case where $D$ and $D^{\prime}$ are not holomorphically equivalent to bounded domains, was treated in [5], [6], and [7], and an affirmative answer to Problem $(*)$ was given for the two-dimensional pseudoconvex case. Besides, arguments in [5], [6], [7] showed that a consideration of Reinhardt domains containing no coordinate hyperplanes has basic importance for the study of Problem $(*)$ in the unbounded case. When dealing with Reinhardt domains containing no coordinate hyperplanes, it seems to be natural to put the assumption that the domains are pseudoconvex. In fact, for example, to each pseudoconvex Reinhardt domain $D$ in $\left(\mathbf{C}^{*}\right)^{n}$, there is a naturally associated integer $\ell(D)$ between 0 and $n$ which indicates the measure of the unboundedness of $D$, and it holds that $D$ is algebraically equivalent to a bounded Reinhardt domain precisely when $\ell(D)=0$ (see Section 2).

Now, our main result of this paper can be stated as follows:

THEOREM 1.2. If two pseudoconvex Reinhardt domains $D$ and $D^{\prime}$ in $\left(\mathbf{C}^{*}\right)^{n}$ are holomorphically equivalent, then they are algebraically equivalent.

It should be remarked here that if two pseudoconvex Reinhardt domains $D$ and $D^{\prime}$ in $\left(\mathbf{C}^{*}\right)^{n}$ are holomorphically equivalent, then we have $\ell(D)=\ell\left(D^{\prime}\right)$ (see Section 2), and when $\ell(D)=\ell\left(D^{\prime}\right)=0$, Theorem 1.2 is an immediate consequence of [3, Section 4, Theorem 1], because $D$ and $D^{\prime}$ are algebraically equivalent to bounded Reinhardt domains.

The proof of Theorem 1.2 will be given in Section 3 after making some preparations in Section 2. Also, in Section 4, we present an application of Theorem 1.2 to the conjugacy of torus actions.

2. Some observations. There is a useful correspondence between Reinhardt domains and tube domains. A tube domain $T_{\Omega}$ in $\mathbf{C}^{n}$ is a domain in $\mathbf{C}^{n}$ given by $T_{\Omega}=\Omega+\sqrt{-1} \mathbf{R}^{n}$, where $\Omega$ is a domain in $\mathbf{R}^{n}$. We call $\Omega$ the base of $T_{\Omega}$. For each element $\eta$ of $\mathbf{R}^{n}$, we define 
an automorphism $\sigma_{\eta}$ of $T_{\Omega}$ given as a translation of $\mathbf{C}^{n}$ by $\sigma_{\eta}(\zeta)=\zeta+\sqrt{-1} \eta$ for $\zeta \in T_{\Omega}$. Now consider a mapping ord : $\left(\mathbf{C}^{*}\right)^{n} \rightarrow \mathbf{R}^{n}$ defined by

$$
\operatorname{ord}\left({ }^{t}\left(z_{1}, \ldots, z_{n}\right)\right)={ }^{t}\left(-\frac{1}{2 \pi} \log \left|z_{1}\right|, \ldots,-\frac{1}{2 \pi} \log \left|z_{n}\right|\right) \text { for }{ }^{t}\left(z_{1}, \ldots, z_{n}\right) \in\left(\mathbf{C}^{*}\right)^{n}
$$

If $D$ is a Reinhardt domain in $\left(\mathbf{C}^{*}\right)^{n}$, then ord $(D)$ is a domain in $\mathbf{R}^{n}$, and it is well known that $D$ is pseudoconvex if and only if $\operatorname{ord}(D)$ is a convex domain in $\mathbf{R}^{n}$. To each Reinhardt domain $D$ in $\left(\mathbf{C}^{*}\right)^{n}$, there is associated a tube domain $T_{\Omega}$ in $\mathbf{C}^{n}$ with $\Omega=\operatorname{ord}(D)$. The tube domain $T_{\Omega}$ naturally becomes a covering manifold of $D$. Indeed, introduce a covering $\varpi: \mathbf{C}^{n} \rightarrow\left(\mathbf{C}^{*}\right)^{n}$ defined by

$$
\varpi\left({ }^{t}\left(\zeta_{1}, \ldots, \zeta_{n}\right)\right)={ }^{t}\left(e^{-2 \pi \zeta_{1}}, \ldots, e^{-2 \pi \zeta_{n}}\right) \quad \text { for }{ }^{t}\left(\zeta_{1}, \ldots, \zeta_{n}\right) \in \mathbf{C}^{n}
$$

Then we have $T_{\Omega}=\varpi^{-1}(D)$, and the restriction $\varpi: T_{\Omega} \rightarrow D$ is the covering projection. The covering transformation group for $\varpi: T_{\Omega} \rightarrow D$ is given by $\left\{\sigma_{\eta} ; \eta \in \mathbf{Z}^{n}\right\}$. We call $T_{\Omega}$ the covering tube domain of $D$ and $\varpi: T_{\Omega} \rightarrow D$ the canonical covering projection. Note that if $D$ is pseudoconvex, then $\varpi: T_{\Omega} \rightarrow D$ gives the universal covering of $D$. Indeed, if $D$ is pseudoconvex, then $T_{\Omega}$ is simply connected, since $\Omega$ is convex.

Let $D$ be a pseudoconvex Reinhardt domain in $\left(\mathbf{C}^{*}\right)^{n}$ and write $\Omega=\operatorname{ord}(D)$. Since $\Omega$ is convex, there exists an affine transformation $f$ of $\mathbf{R}^{n}$ such that

$$
f(\Omega)=\Xi^{(1)} \times \mathbf{R}^{l},
$$

where $l$ is an integer between 0 and $n$ and $\Xi^{(1)}$ is a convex domain in $\mathbf{R}^{n-l}$ containing no complete straight lines (cf. [2, Section 1.4]). This implies that if, for each point $\xi$ of $\Omega$, we denote by $V_{\xi}$ the maximal vector subspace of $\mathbf{R}^{n}$ such that $\xi+V_{\xi} \subset \Omega$, then the vector subspaces $V_{\xi}, \xi \in \Omega$ coincide with each other, and its dimension is equal to $l$. As a consequence, $l$ is independent of the choice of $f$ satisfying (2.1). Therefore the integer $l$ is an invariant associated with $D$, which we denote by $\ell(D)$.

Here are some observations about $\ell(D)$. Let $D$ be a pseudoconvex Reinhardt domain in $\left(\mathbf{C}^{*}\right)^{n}$ and write $\Omega=\operatorname{ord}(D)$. When $\ell(D)=0$, the domain $D$ is algebraically equivalent to a bounded Reinhardt domain in $\left(\mathbf{C}^{*}\right)^{n}$ (see [2, Lemma 1.4.12]). On the other hand, when $\ell(D)>0$, write an affine transformation $f$ of $\mathbf{R}^{n}$ satisfying $(2.1)$ as $f(\xi)=L \xi+b$ for $\xi \in \mathbf{R}^{n}$, where $L \in G L(n, \mathbf{R})$ and $b \in \mathbf{R}^{n}$. If we define an affine transformation $F$ of $\mathbf{C}^{n}$ by $F(\zeta)=L \zeta+b$ for $\zeta \in \mathbf{C}^{n}$, then we have

$$
F\left(T_{\Omega}\right)=T_{f(\Omega)}=T_{\Xi^{(1)} \times \mathbf{R}^{\ell(D)}}=T_{\Xi^{(1)}} \times T_{\mathbf{R}^{\ell(D)}}=T_{\Xi^{(1)}} \times \mathbf{C}^{\ell(D)},
$$

and hence $T_{\Omega}$ is holomorphically equivalent to $T_{\Xi^{(1)}} \times \mathbf{C}^{\ell(D)}$. Since $T_{\Omega}$ is a covering manifold of $D$, this shows that $D$ admits a nonconstant holomorphic mapping from $\mathbf{C}^{\ell(D)}$. Note that $\ell(D)=n$ if and only if $D=\left(\mathbf{C}^{*}\right)^{n}$.

The following lemma implies that $\ell(D)$ is a biholomorphic invariant. 
LEMma 2.1. Let $E \times \mathbf{C}^{l}$ and $E^{\prime} \times \mathbf{C}^{l^{\prime}}$ be two domains in $\mathbf{C}^{n}$, where both $E \subset \mathbf{C}^{n-l}$ and $E^{\prime} \subset \mathbf{C}^{n-l^{\prime}}$ are holomorphically equivalent to bounded domains. If there exists a biholomorphic mapping $\Phi: E \times \mathbf{C}^{l} \rightarrow E^{\prime} \times \mathbf{C}^{l^{\prime}}$. then $l$ is equal to $l^{\prime}$. Moreover, if we write each point $w \in \mathbf{C}^{n}=\mathbf{C}^{n-l} \times \mathbf{C}^{l}$ as

$$
w=\left(\begin{array}{l}
w^{(1)} \\
w^{(2)}
\end{array}\right), \quad w^{(1)} \in \mathbf{C}^{n-l}, \quad w^{(2)} \in \mathbf{C}^{l},
$$

then $\Phi$ is of the form

$$
\Phi: E \times \mathbf{C}^{l} \ni w=\left(\begin{array}{c}
w^{(1)} \\
w^{(2)}
\end{array}\right) \longmapsto\left(\begin{array}{c}
\Phi^{(1)}\left(w^{(1)}\right) \\
\Phi^{(2)}(w)
\end{array}\right) \in E^{\prime} \times \mathbf{C}^{l^{\prime}}
$$

for a biholomorphic mapping $\Phi^{(1)}: E \rightarrow E^{\prime}$.

Proof. We define $\Sigma_{w^{(1)}}=\left\{w^{(1)}\right\} \times \mathbf{C}^{l}$ for $w^{(1)} \in E$. Since $E$ is holomorphically equivalent to a bounded domain, the collection $\left\{\Sigma_{w^{(1)}}\right\}_{w^{(1)} \in E}$ is the Liouville foliation on

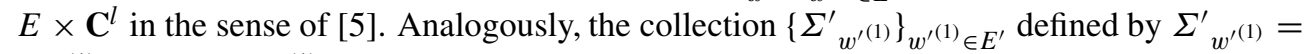
$\left\{w^{\prime(1)}\right\} \times \mathbf{C}^{l^{\prime}}$ for $w^{\prime(1)} \in E^{\prime}$ is the Liouville foliation on $E^{\prime} \times \mathbf{C}^{\prime^{\prime}}$. Note that $\Sigma_{w^{(1)}}$ and $\Sigma^{\prime}{ }_{w^{\prime}}(1)$ are holomorphically equivalent to $\mathbf{C}^{l}$ and $\mathbf{C}^{l^{\prime}}$, respectively. By [5, Proposition 2.1], there exists a bijection $\tau: E \rightarrow E^{\prime}$ such that

$$
\Phi\left(\Sigma_{w^{(1)}}\right)=\Sigma_{\tau\left(w^{(1)}\right)}^{\prime} \quad \text { for every } w^{(1)} \in E .
$$

As a consequence, $\Phi$ induces a biholomorphic mapping from $\Sigma_{w^{(1)}}$ to $\Sigma_{\tau\left(w^{(1)}\right)}^{\prime}$, which implies the first assertion. The second assertion also follows from (2.2).

COROllary 2.2. If two pseudoconvex Reinhardt domains $D$ and $D^{\prime}$ in $\left(\mathbf{C}^{*}\right)^{n}$ are holomorphically equivalent, then $\ell(D)$ is equal to $\ell\left(D^{\prime}\right)$.

Proof. Let $T_{\Omega}$ and $T_{\Omega^{\prime}}$ be the covering tube domains of $D$ and $D^{\prime}$, respectively. Since $D$ and $D^{\prime}$ are pseudoconvex, $\varpi: T_{\Omega} \rightarrow D$ and $\varpi: T_{\Omega^{\prime}} \rightarrow D^{\prime}$ are the universal coverings of $D$ and $D^{\prime}$, respectively. Since $D$ and $D^{\prime}$ are holomorphically equivalent, $T_{\Omega}$ and $T_{\Omega^{\prime}}$ are also holomorphically equivalent. On the other hand, as observed before Lemma $2.1, T_{\Omega}$ is holomorphically equivalent to $T_{\Xi^{(1)}} \times \mathbf{C}^{\ell(D)}$, where $T_{\Xi^{(1)}}$ is a tube domain in $\mathbf{C}^{n-\ell(D)}$ whose base $\Xi^{(1)}$ is a convex domain in $\mathbf{R}^{n-\ell(D)}$ containing no complete straight lines, while $T_{\Omega^{\prime}}$ is holomorphically equivalent to $T_{\Xi^{\prime}(1)} \times \mathbf{C}^{\ell\left(D^{\prime}\right)}$, where $T_{\Xi^{\prime}}$ is is a tube domain in $\mathbf{C}^{n-\ell\left(D^{\prime}\right)}$ whose base $\Xi^{\prime(1)}$ is a convex domain in $\mathbf{R}^{n-\ell\left(D^{\prime}\right)}$ containing no complete straight lines. We thus conclude that $T_{\Xi^{(1)}} \times \mathbf{C}^{\ell(D)}$ and $T_{\Xi^{\prime(1)}} \times \mathbf{C}^{\ell\left(D^{\prime}\right)}$ are holomorphically equivalent. Since both $T_{\Xi^{(1)}}$ and $T_{\Xi^{\prime}}$ are holomorphically equivalent to bounded domains, Lemma 2.1 implies that $\ell(D)=\ell\left(D^{\prime}\right)$.

We denote by $G L(n, \mathbf{Z}) \ltimes \mathbf{C}^{n}$ the group of all complex affine transformations of $\mathbf{C}^{n}$ whose linear parts belong to $G L(n, \mathbf{Z})$. We discuss the relation between $\operatorname{Aut}_{\text {alg }}\left(\left(\mathbf{C}^{*}\right)^{n}\right)$ and $G L(n, \mathbf{Z}) \ltimes \mathbf{C}^{n}$. Let $\Phi$ be any element of $G L(n, \mathbf{Z}) \ltimes \mathbf{C}^{n}$ and write $\Phi(\zeta)=A \zeta+\beta$ for 
$\zeta \in \mathbf{C}^{n}$, where $A=\left(a_{i j}\right) \in G L(n, \mathbf{Z})$ and $\beta=\left(\beta_{i}\right) \in \mathbf{C}^{n}$. Then we can define an element $\varphi$ of $\operatorname{Aut}_{\text {alg }}\left(\left(\mathbf{C}^{*}\right)^{n}\right)$ by

$$
\begin{gathered}
\varphi:\left(\mathbf{C}^{*}\right)^{n} \ni^{t}\left(z_{1}, \ldots, z_{n}\right) \longmapsto{ }^{t}\left(w_{1}, \ldots, w_{n}\right) \in\left(\mathbf{C}^{*}\right)^{n}, \\
w_{i}=e^{-2 \pi \beta_{i}} z_{1}^{a_{i 1}} \cdots z_{n}^{a_{i n}}, \quad i=1, \ldots, n .
\end{gathered}
$$

The mapping $\rho: G L(n, \mathbf{Z}) \ltimes \mathbf{C}^{n} \rightarrow \operatorname{Aut}_{\text {alg }}\left(\left(\mathbf{C}^{*}\right)^{n}\right)$ sending $\Phi$ to $\varphi$ is a group homomorphism. Note that the kernel of $\rho$ is given by $\left\{\sigma_{\eta} ; \eta \in \mathbf{Z}^{n}\right\} \subset \operatorname{Aut}\left(\mathbf{C}^{n}\right)$, and $\rho$ is compatible with the covering projection $\varpi: \mathbf{C}^{n} \rightarrow\left(\mathbf{C}^{*}\right)^{n}$ in the following sense:

$$
\varpi \circ \Phi=\rho(\Phi) \circ \varpi \quad \text { for every } \Phi \in G L(n, \mathbf{Z}) \ltimes \mathbf{C}^{n} .
$$

Let $D$ and $D^{\prime}$ be Reinhardt domains in $\left(\mathbf{C}^{*}\right)^{n}$, and $T_{\Omega}$ and $T_{\Omega^{\prime}}$ the tube domains in $\mathbf{C}^{n}$ given as the covering of $D$ and $D^{\prime}$, respectively. If $\Phi$ is an element of $G L(n, \mathbf{Z}) \ltimes \mathbf{C}^{n}$, then, by (2.3), we have $\rho(\Phi)(D)=D^{\prime}$ precisely when $\Phi\left(T_{\Omega}\right)=T_{\Omega^{\prime}}$. As a consequence, we see that if there exists an element $\Phi$ of $G L(n, \mathbf{Z}) \ltimes \mathbf{C}^{n}$ such that $\Phi\left(T_{\Omega}\right)=T_{\Omega^{\prime}}$, then $D$ and $D^{\prime}$ are algebraically equivalent.

If $\Phi: T_{\Omega} \rightarrow T_{\Omega^{\prime}}$ is a biholomorphic mapping between $T_{\Omega}$ and $T_{\Omega^{\prime}}$ and there exists $A \in G L(n, \mathbf{Z})$ such that

$$
\Phi(\zeta+\sqrt{-1} m)=\Phi(\zeta)+\sqrt{-1} A m \quad \text { for every } \zeta \in T_{\Omega} \text { and every } m \in \mathbf{Z}^{n}
$$

then, since the covering transformation groups for $\varpi: T_{\Omega} \rightarrow D$ and $\varpi^{\prime}: T_{\Omega^{\prime}} \rightarrow D^{\prime}$ are given by $\left\{\sigma_{\eta} ; \eta \in \mathbf{Z}^{n}\right\} \subset \operatorname{Aut}\left(T_{\Omega}\right)$ and $\left\{\sigma_{\eta} ; \eta \in \mathbf{Z}^{n}\right\} \subset \operatorname{Aut}\left(T_{\Omega^{\prime}}\right)$, respectively, it follows that there exists a biholomorphic mapping $\varphi: D \rightarrow D^{\prime}$ with $\varpi^{\prime} \circ \Phi=\varphi \circ \varpi$. Conversely, when $D$ and $D^{\prime}$ are pseudoconvex, every biholomorphic mapping $\varphi: D \rightarrow D^{\prime}$ between $D$ and $D^{\prime}$ has a lifting $\Phi: T_{\Omega} \rightarrow T_{\Omega^{\prime}}$, i.e., a biholomorphic mapping $\Phi: T_{\Omega} \rightarrow T_{\Omega^{\prime}}$ such that $\varpi^{\prime} \circ \Phi=\varphi \circ \varpi$, satisfying (2.4) for some $A \in G L(n, \mathbf{Z})$, because $\varpi: T_{\Omega} \rightarrow D$ and $\varpi^{\prime}: T_{\Omega^{\prime}} \rightarrow D^{\prime}$ are the universal coverings of $D$ and $D^{\prime}$, respectively. As a consequence, we have the following proposition, which is a useful tool in our investigation.

PROPOSITION 2.3. Let $\Phi: T_{\Omega} \rightarrow T_{\Omega^{\prime}}$ be a biholomorphic mapping of tube domains in $\mathbf{C}^{n}$ such that the convex hulls of $\Omega$ and $\Omega^{\prime}$ contain no complete straight lines. Suppose that there exist $A, B \in G L(n, \mathbf{R})$ such that

$$
\Phi(\zeta+\sqrt{-1} A m)=\Phi(\zeta)+\sqrt{-1} B m \text { for every } \zeta \in T_{\Omega} \text { and every } m \in \mathbf{Z}^{n}
$$

Then $\Phi$ is an affine transformation of $\mathbf{C}^{n}$ whose linear part belongs to $G L(n, \mathbf{R})$.

Proof. We define linear automorphisms $F_{A}$ and $F_{B}$ of $\mathbf{C}^{n}$ by $F_{A}(w)=A w$ and $F_{B}(w)$ $=B w$ for $w \in \mathbf{C}^{n}$. Then $F_{A}{ }^{-1}\left(T_{\Omega}\right)$ and $F_{B}{ }^{-1}\left(T_{\Omega^{\prime}}\right)$ are tube domains in $\mathbf{C}^{n}$. Indeed, writing $\Xi=A^{-1} \Omega$ and $\Xi^{\prime}=B^{-1} \Omega^{\prime}$, we have $F_{A}{ }^{-1}\left(T_{\Omega}\right)=T_{\Xi}$ and $F_{B}{ }^{-1}\left(T_{\Omega^{\prime}}\right)=T_{\Xi^{\prime}}$. Note that, since the convex hulls of $\Omega$ and $\Omega^{\prime}$ contain no complete straight lines, so do the convex hulls of $\Xi$ and $\Xi^{\prime}$. We set $E=\varpi\left(T_{\Xi}\right)$ and $E^{\prime}=\varpi\left(T_{\Xi^{\prime}}\right)$. By the definition of $\varpi, E$ and $E^{\prime}$ are Reinhardt domains in $\left(\mathbf{C}^{*}\right)^{n}$, and $T_{\Xi}$ and $T_{\Xi^{\prime}}$ are the covering tube domains of $E$ and $E^{\prime}$, respectively. Applying [3, Section 6, Corollary to Theorem 2] to $E$ and $E^{\prime}$ yields that every biholomorphic mapping of $E$ onto $E^{\prime}$ is induced by an algebraic automorphism of $\left(\mathbf{C}^{*}\right)^{n}$. 
Now consider a biholomorphic mapping $\Psi: T_{\Xi} \rightarrow T_{\Xi^{\prime}}$ between $T_{\Xi}$ and $T_{\Xi^{\prime}}$ given by $\Psi=F_{B}{ }^{-1} \circ \Phi \circ F_{A}$. Then we see from (2.5) that $\Psi$ satisfies $\Psi(w+\sqrt{-1} m)=\Psi(w)+$ $\sqrt{-1} m$ for every $w \in T_{\Xi}$ and every $m \in \mathbf{Z}^{n}$. Therefore, as observed above, there exists a biholomorphic mapping $\psi: E \rightarrow E^{\prime}$ such that $\varpi^{\prime} \circ \Psi=\psi \circ \varpi$. By the result of the preceding paragraph, $\psi$ is induced by an algebraic automorphism of $\left(\mathbf{C}^{*}\right)^{n}$. This implies that we can find an element $\Psi_{0}$ of $G L(n, \mathbf{Z}) \ltimes \mathbf{C}^{n}$ such that $\Psi_{0}\left(T_{\Xi}\right)=T_{\Xi^{\prime}}$ and $\varpi^{\prime} \circ \Psi_{0}=\psi \circ \varpi$ on $T_{\Xi}$. Note that both $\Psi: T_{\Xi} \rightarrow T_{\Xi^{\prime}}$ and $\Psi_{0}: T_{\Xi} \rightarrow T_{\Xi^{\prime}}$ are liftings of $\psi$. Replacing $\Psi_{0}$ by $\sigma_{\eta} \circ \Psi_{0}$ for some $\eta \in \mathbf{Z}^{n}$ if necessary, we may assume that $\Psi\left(w_{0}\right)=\Psi_{0}\left(w_{0}\right)$ for a point $w_{0}$ of $T_{\Xi}$. Then we have $\Psi=\Psi_{0}$ by the uniqueness of lifting, i.e., $\Phi=F_{B} \circ \Psi_{0} \circ F_{A}{ }^{-1}$. Since $F_{B} \circ \Psi_{0} \circ F_{A}^{-1}$ is an affine transformation of $\mathbf{C}^{n}$, this completes the proof.

We conclude this section with a lemma from linear algebra.

LeMmA 2.4. Let $U$ be any real $n \times n$ matrix. Then there exists a diagonal matrix $\operatorname{diag}\left(\varepsilon_{1}, \ldots, \varepsilon_{n}\right)$ of degree $n$ with the $i$-th diagonal entry $\varepsilon_{i}= \pm 1$ such that $I_{n}+$ $U \operatorname{diag}\left(\varepsilon_{1}, \ldots, \varepsilon_{n}\right) \in G L(n, \mathbf{R})$.

Proof. Write $I_{n}=\left(e_{1} \cdots e_{n}\right)$, where $\left\{e_{1}, \ldots, e_{n}\right\}$ is the standard basis of $\mathbf{R}^{n}$. Write $U=\left(u_{1} \cdots u_{n}\right)$ with $u_{1}, \ldots, u_{n} \in \mathbf{R}^{n}$. Then the assertion of Lemma 2.4 is equivalent to the assertion that there exist constants $\varepsilon_{j}= \pm 1, j=1, \ldots, n$, such that $n$ elements $e_{1}+\varepsilon_{1} u_{1}, \ldots, e_{n}+\varepsilon_{n} u_{n}$ of $\mathbf{R}^{n}$ are linearly independent. We prove this assertion by induction on $n$.

When $n=1$, our assertion simply inplies that, for any $u \in \mathbf{R}$, we have $1+u \neq 0$ or $1-u \neq 0$. Clearly, this is true.

Suppose $n>1$. Write

$$
e_{1}=\left(\begin{array}{c}
e_{1}^{\prime} \\
0
\end{array}\right), \ldots, e_{n-1}=\left(\begin{array}{c}
e_{n-1}^{\prime} \\
0
\end{array}\right) \quad \text { and } \quad u_{1}=\left(\begin{array}{c}
u_{1}^{\prime} \\
u_{n, 1}
\end{array}\right), \ldots, u_{n-1}=\left(\begin{array}{c}
u_{n-1}^{\prime} \\
u_{n, n-1}
\end{array}\right)
$$

where $\left\{e_{1}^{\prime}, \ldots, e_{n-1}^{\prime}\right\}$ is the standard basis of $\mathbf{R}^{n-1}, u_{1}^{\prime}, \ldots, u_{n-1}^{\prime} \in \mathbf{R}^{n-1}$ and $u_{n, 1}, \ldots, u_{n, n-1}$ $\in \mathbf{R}$. By the induction hypothesis, there exist constants $\varepsilon_{j}= \pm 1, j=1, \ldots, n-1$, such that $n-1$ elements $e_{1}^{\prime}+\varepsilon_{1} u_{1}^{\prime}, \ldots, e_{n-1}^{\prime}+\varepsilon_{n-1} u_{n-1}^{\prime}$ of $\mathbf{R}^{n-1}$ are linearly independent. We observe that $n-1$ elements $e_{1}+\varepsilon_{1} u_{1}, \ldots, e_{n-1}+\varepsilon_{n-1} u_{n-1}$ of $\mathbf{R}^{n}$ are linearly independent. If $n$ elements $e_{1}+\varepsilon_{1} u_{1}, \ldots, e_{n-1}+\varepsilon_{n-1} u_{n-1}, e_{n}+u_{n}$ of $\mathbf{R}^{n}$ are linearly independent, then our assertion holds for $\varepsilon_{n}=1$. Assume that $e_{1}+\varepsilon_{1} u_{1}, \ldots, e_{n-1}+\varepsilon_{n-1} u_{n-1}, e_{n}+u_{n}$ are linearly dependent. Since $e_{1}+\varepsilon_{1} u_{1}, \ldots, e_{n-1}+\varepsilon_{n-1} u_{n-1}$ are linearly independent, it follows that $e_{n}+u_{n}=\lambda_{1}\left(e_{1}+\varepsilon_{1} u_{1}\right)+\cdots+\lambda_{n-1}\left(e_{n-1}+\varepsilon_{n-1} u_{n-1}\right)$ for some $\lambda_{1}, \ldots, \lambda_{n-1} \in \mathbf{R}$, which implies that $e_{n}-u_{n}=2 e_{n}-\lambda_{1}\left(e_{1}+\varepsilon_{1} u_{1}\right)-\cdots-\lambda_{n-1}\left(e_{n-1}+\varepsilon_{n-1} u_{n-1}\right)$. On the other hand, it is readily verified that $n$ elements $e_{1}+\varepsilon_{1} u_{1}, \ldots, e_{n-1}+\varepsilon_{n-1} u_{n-1}, e_{n}$ of $\mathbf{R}^{n}$ are linearly independent. We thus conclude that $e_{1}+\varepsilon_{1} u_{1}, \ldots, e_{n-1}+\varepsilon_{n-1} u_{n-1}, e_{n}-u_{n}$ are linearly independent, i.e., our assertion is valid for $\varepsilon_{n}=-1$. This completes the proof of Lemma 2.4. 
3. Proof of Theorem 1.2. We first note that, to prove Theorem 1.2, we may replace $D$ and $D^{\prime}$ by domains algebraically equivalent to them. Precisely speaking, if we take Reinhardt domains $E$ and $E^{\prime}$ in $\left(\mathbf{C}^{*}\right)^{n}$ which are algebraically equivalent to $D$ and $D^{\prime}$, respectively, then, to prove Theorem 1.2, it is sufficient to prove that $E$ and $E^{\prime}$ are algebraically equivalent.

Now, by assumption, there exists a biholomorphic mapping $\varphi: D \rightarrow D^{\prime}$. In view of Corollary 2.2, we can set $l=\ell(D)=\ell\left(D^{\prime}\right)$. We also set $k=n-l$. If $l=0$, then, as remarked after Theorem 1.2 in Section $1, D$ and $D^{\prime}$ are algebraically equivalent. If $l=n$, then $D=D^{\prime}=\left(\mathbf{C}^{*}\right)^{n}$, and, clearly, $D$ and $D^{\prime}$ are algebraically equivalent. Therefore suppose that $0<l<n$. Replacing $D$ and $D^{\prime}$ by domains algebraically equivalent to them, we may assume that $D \ni{ }^{t}(1, \ldots, 1)$ and $D^{\prime} \ni^{t}(1, \ldots, 1)$. Let $T_{\Omega}$ and $T_{\Omega^{\prime}}$ denote the covering tube domains of $D$ and $D^{\prime}$, respectively. Note that $\Omega \ni{ }^{t}(0, \ldots, 0)$ and $\Omega^{\prime} \ni{ }^{t}(0, \ldots, 0)$. Let $\tilde{\varphi}: T_{\Omega} \rightarrow T_{\Omega^{\prime}}$ be a biholomorphic mapping given as a lifting of $\varphi: D \rightarrow D^{\prime}$. Then $\tilde{\varphi}$ satisfies the condition that, for some $A \in G L(n, \mathbf{Z})$, we have

$$
\begin{aligned}
\tilde{\varphi}(\zeta+\sqrt{-1} m)= & \tilde{\varphi}(\zeta)+\sqrt{-1} A m \\
& \text { for every } \zeta \in T_{\Omega} \text { and every } m \in \mathbf{Z}^{n} .
\end{aligned}
$$

We may assume that $A=I_{n}$ in (3.1). To see this, write $A^{-1}=\left(b_{i j}\right)$. Since $A^{-1} \in$ $G L(n, \mathbf{Z})$, we can define an algebraic automorphism $\psi$ of $\left(\mathbf{C}^{*}\right)^{n}$ by

$$
\begin{gathered}
\psi:\left(\mathbf{C}^{*}\right)^{n} \ni^{t}\left(z_{1}, \ldots, z_{n}\right) \longmapsto{ }^{t}\left(w_{1}, \ldots, w_{n}\right) \in\left(\mathbf{C}^{*}\right)^{n}, \\
w_{i}=z_{1}^{b_{i 1}} \cdots z_{n}^{b_{i n}}, \quad i=1, \ldots, n .
\end{gathered}
$$

Let $D^{\prime \prime}$ be a Reinhardt domain in $\left(\mathbf{C}^{*}\right)^{n}$ given by $D^{\prime \prime}=\psi\left(D^{\prime}\right)$ and $T_{\Omega^{\prime \prime}}$ the covering tube domain of $D^{\prime \prime}$. By the definition of $\psi$, the mapping $\psi: D^{\prime} \rightarrow D^{\prime \prime}$ has a lifting $\tilde{\psi}: T_{\Omega^{\prime}} \rightarrow$ $T_{\Omega^{\prime \prime}}$ given by

$$
\tilde{\psi}(\zeta)=A^{-1} \zeta \quad \text { for } \zeta \in T_{\Omega^{\prime}}
$$

Also, since $D^{\prime} \ni{ }^{t}(1, \ldots, 1)$ and $\psi\left({ }^{t}(1, \ldots, 1)\right)={ }^{t}(1, \ldots, 1)$, we have $D^{\prime \prime} \ni{ }^{t}(1, \ldots, 1)$. Now, as noted at the beginning of this section, to prove Theorem 1.2, we have only to prove that $D$ and $D^{\prime \prime}$ are algebraically equivalent. Then, for the biholomorphic mapping $\psi \circ \varphi$ : $D \rightarrow D^{\prime \prime}$, its lifting $\widetilde{\psi \circ \varphi}: T_{\Omega} \rightarrow T_{\Omega^{\prime \prime}}$ satisfies

$$
\begin{aligned}
\widetilde{\psi \circ \varphi}(\zeta+\sqrt{-1} m)= & \widetilde{\psi \circ \varphi(\zeta)}+\sqrt{-1} m \\
& \text { for every } \zeta \in T_{\Omega} \text { and every } m \in \mathbf{Z}^{n},
\end{aligned}
$$

because of (3.1), (3.2), and the fact that $\widetilde{\psi \circ \varphi}(\zeta)=\tilde{\psi} \circ \tilde{\varphi}(\zeta)$ for $\zeta \in T_{\Omega}$. Therefore we may replace $(3.1)$ by

$$
\begin{aligned}
\tilde{\varphi}(\zeta+\sqrt{-1} m)= & \tilde{\varphi}(\zeta)+\sqrt{-1} m \\
& \text { for every } \zeta \in T_{\Omega} \text { and every } m \in \mathbf{Z}^{n}
\end{aligned}
$$


For further discussion, we need a method of simultaneous permutation of coordinates. To explain this, let $\tau$ be a permutation of the set $\{1, \ldots, n\}$. We define an algebraic automorphism $f_{\tau}$ of $\left(\mathbf{C}^{*}\right)^{n}$ given as a permutation of coordinates by

$$
\begin{gathered}
f_{\tau}:\left(\mathbf{C}^{*}\right)^{n} \ni{ }^{t}\left(z_{1}, \ldots, z_{n}\right) \longmapsto{ }^{t}\left(w_{1}, \ldots, w_{n}\right) \in\left(\mathbf{C}^{*}\right)^{n}, \\
w_{i}=z_{\tau(i)}, \quad i=1, \ldots, n .
\end{gathered}
$$

Clearly, $f_{\tau}:\left(\mathbf{C}^{*}\right)^{n} \rightarrow\left(\mathbf{C}^{*}\right)^{n}$ has a lifting $F_{\tau}: \mathbf{C}^{n} \rightarrow \mathbf{C}^{n}$ given by

$$
\begin{gathered}
F_{\tau}: \mathbf{C}^{n} \ni{ }^{t}\left(\zeta_{1}, \ldots, \zeta_{n}\right) \longmapsto{ }^{t}\left(\omega_{1}, \ldots, \omega_{n}\right) \in \mathbf{C}^{n}, \\
\omega_{i}=\zeta_{\tau(i)}, \quad i=1, \ldots, n .
\end{gathered}
$$

Note that $F_{\tau}\left(T_{\Omega}\right)$ and $F_{\tau}\left(T_{\Omega^{\prime}}\right)$ are the covering tube domains of $f_{\tau}(D)$ and $f_{\tau}\left(D^{\prime}\right)$, respectively. Now consider a biholomorphic mapping $f_{\tau} \circ \varphi \circ f_{\tau}^{-1}: f_{\tau}(D) \rightarrow f_{\tau}\left(D^{\prime}\right)$ between Reinhardt domains $f_{\tau}(D)$ and $f_{\tau}\left(D^{\prime}\right)$ in $\left(\mathbf{C}^{*}\right)^{n}$. Then a lifting $\left(f_{\tau} \circ \varphi \circ f_{\tau}^{-1}\right)^{\sim}: F_{\tau}\left(T_{\Omega}\right) \rightarrow$ $F_{\tau}\left(T_{\Omega^{\prime}}\right)$ of $f_{\tau} \circ \varphi \circ f_{\tau}^{-1}: f_{\tau}(D) \rightarrow f_{\tau}\left(D^{\prime}\right)$ is given by

$$
\left(f_{\tau} \circ \varphi \circ f_{\tau}^{-1}\right)^{\sim}(\zeta)=F_{\tau} \circ \tilde{\varphi} \circ F_{\tau}^{-1}(\zeta) \text { for } \zeta \in F_{\tau}\left(T_{\Omega}\right) .
$$

It follows from (3.3) and (3.4) that the lifting $\left(f_{\tau} \circ \varphi \circ f_{\tau}^{-1}\right)^{\sim}: F_{\tau}\left(T_{\Omega}\right) \rightarrow F_{\tau}\left(T_{\Omega^{\prime}}\right)$ satisfies

$$
\begin{aligned}
\left(f_{\tau} \circ \varphi \circ f_{\tau}^{-1}\right)^{\sim}(\zeta+\sqrt{-1} m)= & \left(f_{\tau} \circ \varphi \circ f_{\tau}^{-1}\right)^{\sim}(\zeta)+\sqrt{-1} m \\
& \text { for every } \zeta \in F_{\tau}\left(T_{\Omega}\right) \text { and every } m \in \mathbf{Z}^{n} .
\end{aligned}
$$

This shows that if necessary for our argument, we can take $f_{\tau}(D), f_{\tau}\left(D^{\prime}\right), F_{\tau}\left(T_{\Omega}\right), F_{\tau}\left(T_{\Omega^{\prime}}\right)$, $f_{\tau} \circ \varphi \circ f_{\tau}^{-1}: f_{\tau}(D) \rightarrow f_{\tau}\left(D^{\prime}\right),\left(f_{\tau} \circ \varphi \circ f_{\tau}^{-1}\right)^{\sim}: F_{\tau}\left(T_{\Omega}\right) \rightarrow F_{\tau}\left(T_{\Omega^{\prime}}\right)$ as $D, D^{\prime}, T_{\Omega}, T_{\Omega^{\prime}}$, $\varphi: D \rightarrow D^{\prime}, \tilde{\varphi}: T_{\Omega} \rightarrow T_{\Omega^{\prime}}$ without changing the condition (3.3). We call this procedure a simultaneous permutation of coordinates.

Before proceeding to the next step, we make a few preparations from linear algebra. Let $\left\{v_{1}, \ldots, v_{l}\right\}$ and $\left\{\bar{v}_{1}, \ldots, \bar{v}_{l}\right\}$ be two sets of linearly independen $l$ elements of $\mathbf{R}^{n}$, and let $\left\{v_{1}, \ldots, v_{l}\right\}_{\mathbf{R}}$ and $\left\{\bar{v}_{1}, \ldots, \bar{v}_{l}\right\}_{\mathbf{R}}$ denote the vector subspaces of $\mathbf{R}^{n}$ spanned by $\left\{v_{1}, \ldots, v_{l}\right\}$ and $\left\{\bar{v}_{1}, \ldots, \bar{v}_{l}\right\}$, respectively. We define $n \times l$ matrices $M$ and $\bar{M}$ by $M=\left(v_{1} \cdots v_{l}\right)$ and $\bar{M}=\left(\bar{v}_{1} \ldots \bar{v}_{l}\right)$. Then we have $\left\{v_{1}, \ldots, v_{l}\right\}_{\mathbf{R}}=\left\{\bar{v}_{1}, \ldots, \bar{v}_{l}\right\}_{\mathbf{R}}$ if and only if there exists an element $P$ of $G L(l, \mathbf{R})$ such that $\bar{M}=M P$. Now, let $V$ be an $l$-dimensional vector subspace of $\mathbf{R}^{n}$. For each basis $\left\{v_{1}, \ldots, v_{l}\right\}$ of $V$, we define an $n \times l$ matrix $M$ by $M=\left(v_{1} \cdots v_{l}\right)$. Then $M$ is of rank $l$, that is, there exists an $l$-tuple $i_{1}, \ldots, i_{l}$ of indices with $1 \leq i_{1}<\cdots<i_{l} \leq n$ such that $\operatorname{det} M\left(i_{1}, \ldots, i_{l}\right) \neq 0$. We call $M$ a representative of matrix expressions of $V$ or simply a representative of $V$. By what we stated above, any representative $\bar{M}$ of $V$ is obtained from one representative $M$ of $V$ by $\bar{M}=M P$, where $P \in G L(l, \mathbf{R})$. Note that when $M$ and $\bar{M}$ are two representatives of $V$, for every $l$-tuple $i_{1}, \ldots, i_{l}$ of indices with $1 \leq i_{1}<\cdots<i_{l} \leq n$, we have $\operatorname{det} M\left(i_{1}, \ldots, i_{l}\right) \neq 0$ if and only if $\operatorname{det} \bar{M}\left(i_{1}, \ldots, i_{l}\right) \neq 0$.

Turning to the proof of the theorem, let $V$ denote the maximal $l$-dimensional vector subspace of $\mathbf{R}^{n}$ contained in $\Omega$ and let $M$ be a representative of matrix expressions of $V$. Similarly, let $V^{\prime}$ denote the maximal $l$-dimensional vector subspace of $\mathbf{R}^{n}$ contained in $\Omega^{\prime}$ and let 
$M^{\prime}$ be a representative of matrix expressions of $V^{\prime}$. The following lemma plays an important role in our proof. Note that the proof of the lemma depends crucially on the condition (3.3).

LEMMA 3.1. There exists an $l$-tuple $i_{1}, \ldots, i_{l}$ of indices with $1 \leq i_{1}<\cdots<i_{l} \leq n$ such that $\operatorname{det} M\left(i_{1}, \ldots, i_{l}\right) \neq 0$ and $\operatorname{det} M^{\prime}\left(i_{1}, \ldots, i_{l}\right) \neq 0$.

PROOF. Suppose contrarily that, for every $l$-tuple $i_{1}, \ldots, i_{l}$ of indices with $1 \leq i_{1}<$ $\cdots<i_{l} \leq n$, we have $\operatorname{det} M\left(i_{1}, \ldots, i_{l}\right)=0$ or $\operatorname{det} M^{\prime}\left(i_{1}, \ldots, i_{l}\right)=0$.

Since rank $M^{\prime}=l$, by a suitable simultaneous permutation of coordinates, $M^{\prime}$ has the form

$$
M^{\prime}=\left(\begin{array}{l}
M^{(1)} \\
M^{\prime(2)}
\end{array}\right),
$$

where $M^{\prime(2)}$ is an $l \times l$ matrix and $\operatorname{det} M^{(2)} \neq 0$. Write

$$
M=\left(\begin{array}{l}
M^{(1)} \\
M^{(2)}
\end{array}\right),
$$

where $M^{(2)}$ is an $l \times l$ matrix. Since $\operatorname{det} M^{\prime}(k+1, \ldots, n)=\operatorname{det} M^{\prime(2)} \neq 0$, our hypothesis yields that $0=\operatorname{det} M(k+1, \ldots, n)=\operatorname{det} M^{(2)}$, or rank $M^{(2)}<l$. We set $r=l-\operatorname{rank} M^{(2)}$, and write

$$
M^{(2)}=\left(\begin{array}{ll}
M_{11}^{(2)} & M_{12}^{(2)} \\
M_{21}^{(2)} & M_{22}^{(2)}
\end{array}\right),
$$

where $M_{22}^{(2)}$ is an $r \times r$ matrix. By taking another representative of $V$ as $M$ if necessary, we have

$$
\operatorname{rank}\left(\begin{array}{c}
M_{11}^{(2)} \\
M_{21}^{(2)}
\end{array}\right)=l-r .
$$

Therefore we may assume that $\operatorname{det} M_{11}^{(2)} \neq 0$ in (3.6) after a suitable simultaneous permutation of coordinates from the $k+1$-th through the $n$-th. Then, again by taking another representative of $V$ as $M$ if necessary, we have $M_{11}^{(2)}=I_{l-r}$ and $M_{12}^{(2)}=O$. Since rank $M^{(2)}=l-r$, it follows that $M_{22}^{(2)}=O$, so that

$$
M^{(2)}=\left(\begin{array}{ll}
I_{l-r} & O \\
M_{21}^{(2)} & O
\end{array}\right) .
$$

Note that $M^{\prime}$ still has the form (3.5) with $\operatorname{det} M^{\prime(2)} \neq 0$.

We look into $M^{(1)}$. Since rank $M=l$, by a suitable simultaneous permutation of coordinates from the first through the $k$-th, $M^{(1)}$ has the form

$$
M^{(1)}=\left(\begin{array}{ll}
M_{11}^{(1)} & M_{12}^{(1)} \\
M_{21}^{(1)} & M_{22}^{(1)}
\end{array}\right),
$$


where $M_{22}^{(1)}$ is an $r \times r$ matrix and

$$
\operatorname{det}\left(\begin{array}{cc}
M_{21}^{(1)} & M_{22}^{(1)} \\
I_{l-r} & O
\end{array}\right) \neq 0 .
$$

Since det $M_{22}^{(1)} \neq 0$ as a consequence of (3.7), by taking another representative of $V$ as $M$ if necessary, we have $M_{22}^{(1)}=I_{r}$ and $M_{21}^{(1)}=O$. Moreover, again by taking another representative of $V$ as $M$, we conclude that $M$ has the form

$$
M=\left(\begin{array}{cc}
M_{12}^{(1)} & M_{11}^{(1)} \\
I_{r} & O \\
O & I_{l-r} \\
O & M_{21}^{(2)}
\end{array}\right)
$$

Note that we have necessarily $k \geq r$. We set $s=k-r$. Note also that $M^{\prime}$ still has the form (3.5) with $\operatorname{det} M^{\prime(2)} \neq 0$.

We look into $M^{\prime}$. Since $\operatorname{det} M^{\prime(2)} \neq 0$, by taking another representative of $V^{\prime}$ as $M^{\prime}$ if necessary, we have $M^{\prime(2)}=I_{l}$. Write

$$
M^{\prime}=\left(\begin{array}{cc}
M_{11}^{\prime(1)} & M_{12}^{\prime(1)} \\
M_{21}^{\prime(1)} & M_{22}^{\prime(1)} \\
I_{l-r} & O \\
O & I_{r}
\end{array}\right)
$$

where $M_{22}^{\prime(1)}$ is an $r \times r$ matrix. Since $\operatorname{det} M(s+1, \ldots, n-r) \neq 0$ by (3.8), our hypothesis yields that $\operatorname{det} M^{\prime}(s+1, \ldots, n-r)=0$. Therefore, in (3.9), we have

$$
\operatorname{det}\left(\begin{array}{cc}
M_{21}^{\prime(1)} & M_{22}^{\prime(1)} \\
I_{l-r} & O
\end{array}\right)=0 .
$$

This implies that $\operatorname{det} M_{22}^{\prime(1)}=0$.

We now define elements $L$ and $L^{\prime}$ of $G L(n, \mathbf{R})$ by

$$
L=\left(\begin{array}{cccc}
I_{S} & -M_{12}^{(1)} & -M_{11}^{(1)} & O \\
O & O & -M_{21}^{(2)} & I_{r} \\
O & I_{r} & O & O \\
O & O & I_{l-r} & O
\end{array}\right) \quad \text { and } \quad L^{\prime}=\left(\begin{array}{cccc}
I_{S} & O & -M_{11}^{\prime(1)} & -M_{12}^{(1)} \\
O & I_{r} & -M_{21}^{\prime(1)} & -M_{22}^{\prime(1)} \\
O & O & I_{l-r} & O \\
O & O & O & I_{r}
\end{array}\right)
$$

It is readily verified that

$$
L M=\left(\begin{array}{l}
O \\
I_{l}
\end{array}\right) \quad \text { and } \quad L^{\prime} M^{\prime}=\left(\begin{array}{l}
O \\
I_{l}
\end{array}\right) .
$$

Write $\Xi=L \Omega$ and $\Xi^{\prime}=L^{\prime} \Omega^{\prime}$. Then (3.10) implies that

$$
\Xi=\Xi^{(1)} \times \mathbf{R}^{l} \text { and } \Xi^{\prime}=\Xi^{\prime(1)} \times \mathbf{R}^{l},
$$


where $\Xi^{(1)}$ and $\Xi^{\prime(1)}$ are convex domains in $\mathbf{R}^{k}$ containing no complete straight lines. We define automorphisms $F_{L}$ and $F_{L^{\prime}}$ of $\mathbf{C}^{n}$ given as linear transformations by

$$
F_{L}(\zeta)=L \zeta \quad \text { for } \zeta \in \mathbf{C}^{n} \quad \text { and } \quad F_{L^{\prime}}(\zeta)=L^{\prime} \zeta \quad \text { for } \zeta \in \mathbf{C}^{n}
$$

It follows from (3.11) that

$$
\begin{aligned}
& F_{L}\left(T_{\Omega}\right)=T_{\Xi}=T_{\Xi^{(1)}} \times T_{\mathbf{R}^{l}}=T_{\Xi^{(1)}} \times \mathbf{C}^{l} \\
& \quad \text { and } F_{L^{\prime}}\left(T_{\Omega^{\prime}}\right)=T_{\Xi^{\prime}}=T_{\Xi^{\prime(1)}} \times T_{\mathbf{R}^{l}}=T_{\Xi^{\prime(1)}} \times \mathbf{C}^{l} .
\end{aligned}
$$

Consider a biholomorphic mapping $\Phi: T_{\Xi} \rightarrow T_{\Xi^{\prime}}$ given by $\Phi=F_{L^{\prime}} \circ \tilde{\varphi} \circ F_{L}^{-1}$. Then, by (3.3), $\Phi$ satisfies

$$
\begin{aligned}
\Phi(w+\sqrt{-1} L m)= & \Phi(w)+\sqrt{-1} L^{\prime} m \\
& \text { for every } w \in T_{\Xi} \text { and every } m \in \mathbf{Z}^{n} .
\end{aligned}
$$

On the other hand, because of (3.13), an application of Lemma 2.1 to $\Phi$ yields that if each point $w \in \mathbf{C}^{n}=\mathbf{C}^{k} \times \mathbf{C}^{l}$ is written as

$$
w=\left(\begin{array}{c}
w^{(1)} \\
w^{(2)}
\end{array}\right), \quad w^{(1)} \in \mathbf{C}^{k}, w^{(2)} \in \mathbf{C}^{l},
$$

then $\Phi$ has the form

$$
\begin{aligned}
\Phi: T_{\Xi}=T_{\Xi^{(1)}} \times \mathbf{C}^{l} \ni w & =\left(\begin{array}{c}
w^{(1)} \\
w^{(2)}
\end{array}\right) \\
& \longmapsto\left(\begin{array}{c}
\Phi^{(1)}\left(w^{(1)}\right) \\
\Phi^{(2)}(w)
\end{array}\right) \in T_{\Xi^{\prime(1)}} \times \mathbf{C}^{l}=T_{\Xi^{\prime}},
\end{aligned}
$$

where $\Phi^{(1)}: T_{\Xi^{(1)}} \ni w^{(1)} \mapsto \Phi^{(1)}\left(w^{(1)}\right) \in T_{\Xi^{\prime(1)}}$ gives a biholomorphic mapping of $T_{\Xi^{(1)}}$ onto $T_{\Xi^{\prime}}$. Therefore we see from (3.14) that $\Phi^{(1)}$ satisfies

$$
\Phi^{(1)}\left(w^{(1)}+\sqrt{-1} L^{(1)} m\right)=\Phi^{(1)}\left(w^{(1)}\right)+\sqrt{-1} L^{\prime(1)} m
$$
for every $w^{(1)} \in T_{\Xi^{(1)}}$ and every $m \in \mathbf{Z}^{n}$,

where

$$
L^{(1)}=\left(\begin{array}{cccc}
I_{S} & -M_{12}^{(1)} & -M_{11}^{(1)} & O \\
O & O & -M_{21}^{(2)} & I_{r}
\end{array}\right) \text { and } L^{\prime(1)}=\left(\begin{array}{cccc}
I_{S} & O & -M_{11}^{\prime(1)} & -M_{12}^{\prime(1)} \\
O & I_{r} & -M_{21}^{\prime(1)} & -M_{22}^{\prime(1)}
\end{array}\right) .
$$

Write $L^{(1)}=\left(u_{i j}\right)$ and $L^{\prime(1)}=\left(u_{i j}^{\prime}\right)$. Then we have for $m=\left(m_{1}, \ldots, m_{n}\right) \in \mathbf{Z}^{n}$,

$$
\begin{aligned}
& L^{(1)} m={ }^{t}\left(d_{1}, \ldots, d_{k}\right), \\
& \begin{cases}d_{i}=m_{i}+\sum_{j=s+1}^{k} u_{i j} m_{j}+\sum_{j=k+1}^{n-r} u_{i j} m_{j}, & i=1, \ldots, s, \\
d_{i}=m_{l+i}+\sum_{j=k+1}^{n-r} u_{i j} m_{j}, & i=s+1, \ldots, k,\end{cases}
\end{aligned}
$$


and

$$
\begin{aligned}
& L^{\prime(1)} m={ }^{t}\left(d_{1}^{\prime}, \ldots, d_{k}^{\prime}\right), \\
& \left\{\begin{array}{l}
d_{i}^{\prime}=m_{i}+\sum_{j=k+1}^{n-r} u_{i j}^{\prime} m_{j}+\sum_{j=s+1}^{k} u_{i, l+j}^{\prime} m_{l+j}, \quad i=1, \ldots, s, \\
d_{i}^{\prime}=m_{i}+\sum_{j=k+1}^{n-r} u_{i j}^{\prime} m_{j}+\sum_{j=s+1}^{k} u_{i, l+j}^{\prime} m_{l+j}, \quad i=s+1, \ldots, k .
\end{array}\right.
\end{aligned}
$$

We show that $\Phi^{(1)}$ is an affine transformation of $\mathbf{C}^{k}$ whose linear part belongs to $G L(k, \mathbf{R})$. In (3.16), write

$$
m={ }^{t}\left(m_{1}, \ldots, m_{n}\right) \quad \text { and } \quad m^{(1)}={ }^{t}\left(m_{1}, \ldots, m_{s}, m_{s+1}, \ldots, m_{k}\right),
$$

and put

$$
m_{i}=0 \quad \text { for } i=k+1, \ldots, n-r \quad \text { and } \quad m_{l+i}=\varepsilon_{i} m_{i} \quad \text { for } i=s+1, \ldots, k,
$$

where $\varepsilon_{i}= \pm 1, i=s+1, \ldots, k$. Then (3.17) shows that

$$
L^{(1)} m=\left(\begin{array}{ll}
I_{s} & P \\
O & Q
\end{array}\right) m^{(1)},
$$

where $Q$ is an $r \times r$ matrix given by $Q=\operatorname{diag}\left(\varepsilon_{s+1}, \ldots, \varepsilon_{k}\right)$. On the other hand, (3.18) shows that

$$
L^{\prime(1)} m=\left(\begin{array}{ll}
I_{s} & P^{\prime} \\
O & Q^{\prime}
\end{array}\right) m^{(1)},
$$

where $Q^{\prime}$ is an $r \times r$ matrix given by $Q^{\prime}=I_{r}+N \operatorname{diag}\left(\varepsilon_{s+1}, \ldots, \varepsilon_{k}\right), N$ being some $r \times r$ matrix independent of $\varepsilon_{i}, i=s+1, \ldots, k$. Note that, by Lemma 2.4, a suitable choice of $\varepsilon_{i}, i=s+1, \ldots, k$ yields that $Q^{\prime}$ is a nonsingular $r \times r$ matrix. When $\varepsilon_{i}, i=s+1, \ldots, k$ are chosen so, $k \times k$ matrices $C$ and $C^{\prime}$ given by

$$
C=\left(\begin{array}{ll}
I_{S} & P \\
O & Q
\end{array}\right) \quad \text { and } \quad C^{\prime}=\left(\begin{array}{cc}
I_{S} & P^{\prime} \\
O & Q^{\prime}
\end{array}\right)
$$

are nonsingular, and, by (3.16), (3.19), and (3.20), $\Phi^{(1)}$ satisfies

$$
\begin{aligned}
& \Phi^{(1)}\left(w^{(1)}+\sqrt{-1} C m^{(1)}\right)=\Phi^{(1)}\left(w^{(1)}\right)+\sqrt{-1} C^{\prime} m^{(1)} \\
& \quad \text { for every } w^{(1)} \in T_{\Xi^{(1)}} \text { and every } m^{(1)} \in \mathbf{Z}^{k} .
\end{aligned}
$$

Since the bases $\Xi^{(1)}$ and $\Xi^{\prime(1)}$ of the tube domains $T_{\Xi^{(1)}}$ and $T_{\Xi^{\prime(1)}}$ are convex domains in $\mathbf{R}^{k}$ containing no complete straight lines, it follows from Proposition 2.3 that $\Phi^{(1)}$ is an affine transformation of $\mathbf{C}^{k}$ whose linear part belongs to $G L(k, \mathbf{R})$.

What we have shown in the preceding paragraph implies that $\Phi^{(1)}$ is given by

$$
\Phi^{(1)}\left(w^{(1)}\right)=U w^{(1)}+\beta^{(1)} \text { for } w^{(1)} \in T_{\Xi^{(1)}},
$$

where $U \in G L(k, \mathbf{R})$ and $\beta^{(1)} \in \mathbf{C}^{k}$. In (3.16), write

$$
m={ }^{t}\left(m_{1}, \ldots, m_{n}\right) \text { and } m^{(0)}={ }^{t}\left(m_{1}, \ldots, m_{s}, m_{n-r+1}, \ldots, m_{n}\right),
$$


and put $m_{i}=0$ for $i=s+1, \ldots, n-r$. Then (3.17) shows that

$$
L^{(1)} m=m^{(0)} \text {. }
$$

On the other hand, (3.18) shows that

$$
L^{\prime(1)} m=\left(\begin{array}{cc}
I_{S} & -M_{12}^{\prime(1)} \\
O & -M_{22}^{\prime(1)}
\end{array}\right) m^{(0)}
$$

Note that $\operatorname{det} M_{22}^{\prime(1)}=0$. Therefore a $k \times k$ matrix $D^{\prime}$ given by

$$
D^{\prime}=\left(\begin{array}{cc}
I_{S} & -M_{12}^{\prime(1)} \\
O & -M_{22}^{\prime(1)}
\end{array}\right)
$$

is singular, and, by (3.16), (3.22), and (3.23), $\Phi^{(1)}$ satisfies

$$
\begin{aligned}
& \Phi^{(1)}\left(w^{(1)}+\sqrt{-1} m^{(0)}\right)=\Phi^{(1)}\left(w^{(1)}\right)+\sqrt{-1} D^{\prime} m^{(0)} \\
& \quad \text { for every } w^{(1)} \in T_{\Xi^{(1)}} \text { and every } m^{(0)} \in \mathbf{Z}^{k} .
\end{aligned}
$$

It follows from (3.21) and this relation that $U m^{(0)}=D^{\prime} m^{(0)}$ for every $m^{(0)} \in \mathbf{Z}^{k}$, and hence that $U=D^{\prime}$. Since $D^{\prime}$ is a singular $k \times k$ matrix, this contradicts the fact that $U \in G L(k, \mathbf{R})$, and the proof of Lemma 3.1 is completed.

Lemma 3.1 implies that, after a suitable simultaneous permutation of coordinates, we may assume that $M$ and $M^{\prime}$ have the form

$$
M=\left(\begin{array}{l}
M^{(1)} \\
M^{(2)}
\end{array}\right) \quad \text { and } \quad M^{\prime}=\left(\begin{array}{l}
M^{(1)} \\
M^{\prime(2)}
\end{array}\right),
$$

where $M^{(2)}$ and $M^{\prime(2)}$ are $l \times l$ matrices and satisfy the condition that $\operatorname{det} M^{(2)} \neq 0$ and $\operatorname{det} M^{\prime(2)} \neq 0$. Then, by taking another representatives of $V$ and $V^{\prime}$ as $M$ and $M^{\prime}$ if necessary, we have $M^{(2)}=I_{l}$ and $M^{\prime(2)}=I_{l}$.

We now define elements $L$ and $L^{\prime}$ of $G L(n, \mathbf{R})$ by

$$
L=\left(\begin{array}{cc}
I_{k} & -M^{(1)} \\
O & I_{l}
\end{array}\right) \quad \text { and } \quad L^{\prime}=\left(\begin{array}{cc}
I_{k} & -M^{\prime(1)} \\
O & I_{l}
\end{array}\right) .
$$

It is readily verified that

$$
L M=\left(\begin{array}{l}
O \\
I_{l}
\end{array}\right) \quad \text { and } \quad L^{\prime} M^{\prime}=\left(\begin{array}{l}
O \\
I_{l}
\end{array}\right) .
$$

Write $\Xi=L \Omega$ and $\Xi^{\prime}=L^{\prime} \Omega^{\prime}$. Then (3.24) implies that $\Xi=\Xi^{(1)} \times \mathbf{R}^{l}$ and $\Xi^{\prime}=$ $\Xi^{\prime(1)} \times \mathbf{R}^{l}$, where $\Xi^{(1)}$ and $\Xi^{\prime(1)}$ are convex domains in $\mathbf{R}^{k}$ containing no complete straight lines. We define automorphisms $F_{L}$ and $F_{L^{\prime}}$ of $\mathbf{C}^{n}$ given as linear transformations by (3.12). Then we have (3.13). 
Consider a biholomorphic mapping $\Phi: T_{\Xi} \rightarrow T_{\Xi^{\prime}}$ given by $\Phi=F_{L^{\prime}} \circ \tilde{\varphi} \circ F_{L}{ }^{-1}$. Then, as in the proof of Lemma 3.1, we have (3.14) and (3.15). It follows from (3.14) that the mapping $\Phi^{(1)}$ given in (3.15) satisfies

$$
\begin{aligned}
& \Phi^{(1)}\left(w^{(1)}+\sqrt{-1} L^{(1)} m\right)=\Phi^{(1)}\left(w^{(1)}\right)+\sqrt{-1} L^{\prime(1)} m \\
& \text { for every } w^{(1)} \in T_{\Xi^{(1)}} \text { and every } m \in \mathbf{Z}^{n},
\end{aligned}
$$

where

$$
L^{(1)}=\left(\begin{array}{ll}
I_{k} & -M^{(1)}
\end{array}\right) \text { and } \quad L^{\prime(1)}=\left(\begin{array}{ll}
I_{k} & -M^{\prime(1)}
\end{array}\right) .
$$

We show that $\Phi^{(1)}$ is a translation of $\mathbf{C}^{k}$. In (3.25), write $m={ }^{t}\left(m_{1}, \ldots, m_{n}\right)$ and $m^{(1)}={ }^{t}\left(m_{1}, \ldots, m_{k}\right)$, and put $m_{i}=0$ for $i=k+1, \ldots, n$. Then, by (3.26), we have $L^{(1)} m=m^{(1)}$ and $L^{\prime(1)} m=m^{(1)}$, and hence $\Phi^{(1)}$ satisfies

$$
\begin{aligned}
& \Phi^{(1)}\left(w^{(1)}+\sqrt{-1} m^{(1)}\right)=\Phi^{(1)}\left(w^{(1)}\right)+\sqrt{-1} m^{(1)} \\
& \quad \text { for every } w^{(1)} \in T_{\Xi^{(1)}} \text { and every } m^{(1)} \in \mathbf{Z}^{k} .
\end{aligned}
$$

Since the bases $\Xi^{(1)}$ and $\Xi^{\prime(1)}$ of the tube domains $T_{\Xi^{(1)}}$ and $T_{\Xi^{\prime(1)}}$ are convex domains in $\mathbf{R}^{k}$ containing no complete straight lines, it follows from Proposition 2.3 that $\Phi^{(1)}$ is an affine transformation of $\mathbf{C}^{k}$ whose linear part belongs to $G L(k, \mathbf{R})$, so that $\Phi^{(1)}$ is given by

$$
\Phi^{(1)}\left(w^{(1)}\right)=U w^{(1)}+\beta^{(1)} \text { for } w^{(1)} \in T_{\Xi^{(1)}},
$$

where $U \in G L(k, \mathbf{R})$ and $\beta^{(1)} \in \mathbf{C}^{k}$. Substituting (3.28) into (3.27) yields that $U m^{(1)}=m^{(1)}$ for every $m^{(1)} \in \mathbf{Z}^{k}$, which implies that $U=I_{k}$, or $\Phi^{(1)}$ is a translation of $\mathbf{C}^{k}$.

Since $\Phi^{(1)}$ is given by

$$
\Phi^{(1)}\left(w^{(1)}\right)=w^{(1)}+\beta^{(1)} \text { for } w^{(1)} \in T_{\Xi^{(1)}},
$$

we see from (3.25) that $L^{(1)} m=L^{\prime(1)} m$ for every $m \in \mathbf{Z}^{n}$, which implies that $L^{(1)}=L^{(1)}$. Consequently, we have $L=L^{\prime}$. On the other hand, since $\Phi^{(1)}\left(T_{\Xi^{(1)}}\right)=T_{\Xi^{\prime(1)}}$ and since $\Phi^{(1)}$ is given by (3.29), it follows that if we define a translation $S$ of $\mathbf{C}^{n}$ by $S(w)=w+\beta$ for $w \in \mathbf{C}^{n}$, where ${ }^{t} \beta=\left({ }^{t} \beta^{(1)}, 0, \ldots, 0\right)$, then $S\left(T_{\Xi}\right)=T_{\Xi^{\prime}}$, so that $S\left(F_{L}\left(T_{\Omega}\right)\right)=F_{L^{\prime}}\left(T_{\Omega^{\prime}}\right)$. Therefore we obtain $T_{\Omega^{\prime}}=F_{L^{\prime}}{ }^{-1}\left(S\left(F_{L}\left(T_{\Omega}\right)\right)\right)=F_{L}{ }^{-1}\left(S\left(F_{L}\left(T_{\Omega}\right)\right)\right)$. Since $F_{L}{ }^{-1} \circ S \circ F_{L}$ : $T_{\Omega} \rightarrow T_{\Omega^{\prime}}$ is given by a translation of $\mathbf{C}^{n}$, which is an element of $G L(n, \mathbf{Z}) \ltimes \mathbf{C}^{n}$, we conclude that $D$ and $D^{\prime}$ are algebraically equivalent. This completes the proof of Theorem 1.2.

4. Conjugacy of torus actions. As a closely related problem to Problem $(*)$ stated in Section 1, we have the following problem.

Problem (**). Suppose that $T=(U(1))^{n}$ acts on a Reinhardt domain $D$ in $\mathbf{C}^{n}$ not necessarily by the standard rule stated in Section 1 , but by the abstract rule that to each $\alpha \in T$ is associated a biholomorphic mapping $z \mapsto \alpha \cdot z$ of $D$ onto itself such that:

(1) $\alpha \beta \cdot z=\alpha \cdot(\beta \cdot z) \quad$ for $z \in D$ and $\alpha, \beta \in T$;

(2) the mapping $(\alpha, z) \mapsto \alpha \cdot z$ is a $C^{1}$-mapping of the product manifold $T \times D$ onto $D$. 
Suppose further that $T$ acts effectively on $D$, or the unit element of the group $T$ is the only element of $T$ which leaves each $z \in D$ fixed. If we denote again by $T$ the subgroup of $\operatorname{Aut}(D)$ induced by the action of $T$, then does there exist an element $\varphi$ of $\operatorname{Aut}(D)$ such that $\varphi T \varphi^{-1}=T(D)$ ?

Dealing with, as an illustrative example, a Reinhardt domain whose automorphism group has the structure of a Lie group with respect to the compact-open topology, we explain the connection between Problem (*) and Problem (**). To begin with, we reproduce from [3] a result that an affirmative answer to Problem $(* *)$ is given for such a Reinhardt domain.

THEOREM 4.1. Let D be a Reinhardt domain whose automorphism group $\operatorname{Aut}(D)$ has the structure of a Lie group with respect to the compact-open topology. If $T$ is a torus in the Lie group $\operatorname{Aut}(D)$, that is, a connected compact abelian subgroup of the Lie group $\operatorname{Aut}(D)$, then there exists an element $g$ of $\operatorname{Aut}(D)$ such that $g T g^{-1} \subset T(D)$. Consequently, any $n$ dimensional torus in $\operatorname{Aut}(D)$ is conjugate to $T(D)$ under an inner automorphism of $\operatorname{Aut}(D)$.

SKETCH OF PROOF. The point is the fact that $T(D)$ is a maximal torus in $\operatorname{Aut}(D)$. Indeed, let $T^{\prime}$ be a torus in $\operatorname{Aut}(D)$ containing $T(D)$, and take any element $f={ }^{t}\left(f_{1}, \ldots, f_{n}\right)$ of $T^{\prime}$, where $f_{1}, \ldots, f_{n}$ are holomorphic functions on $D$. Since $f$ commutes with every element of $T(D)$, it follows that the functions $f_{1}, \ldots, f_{n}$ satisfy the condition that, for $i=$ $1, \ldots, n$,

$$
\begin{aligned}
& f_{i}\left({ }^{t}\left(\alpha_{1} z_{1}, \ldots, \alpha_{n} z_{n}\right)\right)=\alpha_{i} f_{i}(z) \\
& \text { for all } z={ }^{t}\left(z_{1}, \ldots, z_{n}\right) \in D \text { and all } \alpha_{1}, \ldots, \alpha_{n} \in U(1) .
\end{aligned}
$$

By the uniqueness of Laurent expansions, we see from this fact that $f$ is given by $f(z)=$ ${ }^{t}\left(\gamma_{1} z_{1}, \ldots, \gamma_{n} z_{n}\right)$ for $z={ }^{t}\left(z_{1}, \ldots, z_{n}\right) \in D$, where $\gamma_{1}, \ldots, \gamma_{n} \in \mathbf{C}^{*}$. If we write $\gamma(f)=$ ${ }^{t}\left(\gamma_{1}, \ldots, \gamma_{n}\right)$, then the mapping $\gamma$ of $T^{\prime}$ into $\left(\mathbf{C}^{*}\right)^{n}$ sending $f$ to $\gamma(f)$ is a continuous group homomorphism between the Lie groups $T^{\prime}$ and $\left(\mathbf{C}^{*}\right)^{n}$. The assumption that $T^{\prime}$ is a torus in Aut $(D)$ containing $T(D)$ implies that $\gamma\left(T^{\prime}\right)$ is a torus in $\left(\mathbf{C}^{*}\right)^{n}$ containing $(U(1))^{n}$. Since $(U(1))^{n}$ is a maximal torus in $\left(\mathbf{C}^{*}\right)^{n}$, we obtain $\gamma\left(T^{\prime}\right)=(U(1))^{n}$, and therefore $T^{\prime}=T(D)$, which shows that $T(D)$ is a maximal torus in $\operatorname{Aut}(D)$. Once we know that $T(D)$ is a maximal torus in $\operatorname{Aut}(D)$, the assertion of Theorem 4.1 is an immediate consequence of the conjugacy theorems in the theory of Lie groups.

By a well-known theorem of $\mathrm{H}$. Cartan, if a Reinhardt domain $D$ in $\mathbf{C}^{n}$ is holomorphically equivalent to a bounded domain, then $\operatorname{Aut}(D)$ has the structure of a Lie group with respect to the compact-open topology, and hence Theorem 4.1 applies. We observe that the above proof of Theorem 4.1 relies heavily on the fact that $\operatorname{Aut}(D)$ is small in the sense that $\operatorname{Aut}(D)$ is a space of finite dimension. Therefore, when $\operatorname{Aut}(D)$ is not small, another approach to Problem $(* *)$ is needed. Actually, if $D$ is not holomorphically equivalent to a bounded domain, then $\operatorname{Aut}(D)$ is in general very big, and far from being of finite dimension.

As an immediate consequence of Theorem 4.1, we obtain an affirmative answer to Problem $(*)$ for Reinhardt domains as in Theorem 4.1 (cf. [3, Section 4, Theorem 1]). Indeed, let $D$ and $D^{\prime}$ be two Reinhardt domains in $\mathbf{C}^{n}$ whose automorphism groups $\operatorname{Aut}(D)$ and 
Aut $\left(D^{\prime}\right)$ have the structure of Lie groups with respect to the compact-open topology. If $D$ and $D^{\prime}$ are holomorphically equivalent, and if $\varphi: D \rightarrow D^{\prime}$ is a biholomorphic mapping between $D$ and $D^{\prime}$, then $\varphi T(D) \varphi^{-1}$ is an $n$-dimensional torus in $\operatorname{Aut}\left(D^{\prime}\right)$, and hence an application of Theorem 4.1 to $T\left(D^{\prime}\right)$ and $\varphi T(D) \varphi^{-1}$ yields that $T\left(D^{\prime}\right)=g\left(\varphi T(D) \varphi^{-1}\right) g^{-1}=$ $(g \circ \varphi) T(D)(g \circ \varphi)^{-1}$ for some $g \in \operatorname{Aut}\left(D^{\prime}\right)$. By Proposition 1.1, we see from this fact that the biholomorphic mapping $g \circ \varphi: D \rightarrow D^{\prime}$ between $D$ and $D^{\prime}$ is induced by an algebraic automorphism of $\left(\mathbf{C}^{*}\right)^{n}$, or $D$ and $D^{\prime}$ are algebraically equivalent.

Now, consider Problem (**) for the unbounded case where a Reinhardt domain $D$ in $\mathbf{C}^{n}$ is not holomorphically equivalent to a bounded domain. The case of $D=\mathbf{C}^{k} \times\left(\mathbf{C}^{*}\right)^{n-k}$ was treated in Barrett, Bedford, and Dadok [1]. On the other hand, combined with a result of [1], Theorem 1.2 has the following consequence.

THEOREM 4.2. Let D be a pseudoconvex Reinhardt domain in $\left(\mathbf{C}^{*}\right)^{n}$ with $0<\ell(D)<$ $n$. Suppose that a subgroup $T$ of $\operatorname{Aut}(D)$ is given as in Problem (**). Then there exists an element $\varphi$ of $A u t(D)$ such that $\varphi T \varphi^{-1}=T(D)$.

Proof. By [1], there exists a biholomorphic mapping $\psi$ of $D$ onto a Reinhardt domain $D^{\prime}$ in $\mathbf{C}^{n}$ such that

$$
\psi T \psi^{-1}=T\left(D^{\prime}\right) .
$$

Then $D^{\prime}$ is contained in $\left(\mathbf{C}^{*}\right)^{n}$, because the pseudoconvex Reinhardt domains $D$ and $D^{\prime}$ must be homeomorphic. Note that $D^{\prime}$ satisfies $0<\ell\left(D^{\prime}\right)<n$. Since $D$ and $D^{\prime}$ are holomorphically equivalent, we see by Theorem 1.2 that there exists an algebraic automorphism $\theta$ of $\left(\mathbf{C}^{*}\right)^{n}$ such that $\theta(D)=D^{\prime}$. Then we have

$$
\theta T(D) \theta^{-1}=T\left(D^{\prime}\right) .
$$

It follows from (4.1) and (4.2) that $\psi T \psi^{-1}=T\left(D^{\prime}\right)=\theta T(D) \theta^{-1}$, and hence that $\left(\theta^{-1} \circ\right.$ $\psi) T\left(\theta^{-1} \circ \psi\right)^{-1}=T(D)$. Since $\theta^{-1} \circ \psi$ is a biholomorphic mapping of $D$ onto $D$, or an automorphism of $D$, this concludes the assertion of Theorem 4.2.

5. A remark on automorphisms of unbounded Reinhardt domains. We first observe that if $\varphi: D \rightarrow D^{\prime}$ is a biholomorphic mapping between two Reinhardt domains $D$ and $D^{\prime}$ in $\left(\mathbf{C}^{*}\right)^{n}$, and if it has a lifting $\tilde{\varphi}: T_{\Omega} \rightarrow T_{\Omega^{\prime}}$, where $T_{\Omega}$ and $T_{\Omega^{\prime}}$ are the covering tube domains of $D$ and $D^{\prime}$, respectively, then an element $A$ of $G L(n, \mathbf{Z})$ for which we have

$$
\tilde{\varphi}(\zeta+\sqrt{-1} m)=\tilde{\varphi}(\zeta)+\sqrt{-1} A m \quad \text { for every } \zeta \in T_{\Omega} \text { and every } m \in \mathbf{Z}^{n}
$$

is uniquely determined by $\varphi$ independently of the choice of the lifting $\tilde{\varphi}$. We denote this element $A$ of $G L(n, \mathbf{Z})$ by $A(\varphi)$.

Now, for a pseudoconvex Reinhardt domain $D$ in $\left(\mathbf{C}^{*}\right)^{n}$ with $\ell(D)>0$, we define a subgroup $\operatorname{Aut}(D)^{\circ}$ of $\operatorname{Aut}(D)$ by

$$
\operatorname{Aut}(D)^{\circ}=\left\{\varphi \in \operatorname{Aut}(D) ; A(\varphi)=I_{n}\right\} .
$$

In this section, by making use of our argument in the proof of Theorem 1.2, we give a description of automorphisms of $D$ belonging to $\operatorname{Aut}(D)^{\circ}$ when $0<\ell(D)<n$. 
Let $D$ be a pseudoconvex Reinhardt domain in $\left(\mathbf{C}^{*}\right)^{n}$ with $0<\ell(D)<n$ and write $l=\ell(D)$ and $k=n-l$. We denote by $T_{\Omega}$ the covering tube domain of $D$ and assume that $\Omega$ contains the origin. Let $V$ denote the maximal $l$-dimensional vector subspace of $\mathbf{R}^{n}$ contained in $\Omega$ and let $M$ be a representative of matrix expressions of $V$. After a suitable permutation of coordinates, we may assume that $M$ has the form

$$
M=\left(\begin{array}{l}
M^{(1)} \\
M^{(2)}
\end{array}\right),
$$

where $M^{(2)}$ is an $l \times l$ matrix and satisfies $\operatorname{det} M^{(2)} \neq 0$. Then, by taking another representative of $V$ as $M$ if necessary, we have $M^{(2)}=I_{l}$. We define an element $L$ of $G L(n, \mathbf{R})$ by

$$
L=\left(\begin{array}{cc}
I_{k} & -M^{(1)} \\
O & I_{l}
\end{array}\right),
$$

and write $\Xi=L \Omega$. Then we have $\Xi=\Xi^{(1)} \times \mathbf{R}^{l}$, where $\Xi^{(1)}$ is a convex domain in $\mathbf{R}^{k}$ containing no complete straight lines. Therefore, for an automorphism $F_{L}$ of $\mathbf{C}^{n}$ defined by $F_{L}(\zeta)=L \zeta$ for $\zeta \in \mathbf{C}^{n}$, we have $F_{L}\left(T_{\Omega}\right)=T_{\Xi}=T_{\Xi^{(1)}} \times \mathbf{C}^{l}$.

Let $\varphi$ be any element of $\operatorname{Aut}(D)^{\circ}$. Let $\tilde{\varphi}$ be an automorphism of $T_{\Omega}$ given as a lifting of $\varphi$ and set $\Phi=F_{L} \circ \tilde{\varphi} \circ F_{L}{ }^{-1}$. Then, by the condition $A(\varphi)=I_{n}$, the automorphism $\Phi$ of $T_{\Xi}$ satisfies (3.14) with $T_{\Xi^{\prime}}=T_{\Xi}$ and $L^{\prime}=L$. On the other hand, $\Phi$ has the form (3.15) with $T_{\Xi^{\prime}}=T_{\Xi}$ and $T_{\Xi^{\prime}}=T_{\Xi^{(1)}}$, where $\Phi^{(1)}: T_{\Xi^{(1)}} \ni w^{(1)} \mapsto \Phi^{(1)}\left(w^{(1)}\right) \in T_{\Xi^{(1)}}$ gives an automorphism of $T_{\Xi^{(1)}}$, while, for each fixed $w^{(1)} \in T_{\Xi^{(1)}}$, the mapping

$$
\mathbf{C}^{l} \ni w^{(2)} \mapsto \Phi^{(2)}\left(\begin{array}{l}
w^{(1)} \\
w^{(2)}
\end{array}\right) \in \mathbf{C}^{l}
$$

gives an automorphism of $\mathbf{C}^{l}$. As a consequence of (3.14) with $T_{\Xi^{\prime}}=T_{\Xi}$ and $L^{\prime}=L$, the mapping $\Phi^{(1)}$ satisfies (3.25) with $T_{\Xi^{\prime}(1)}=T_{\Xi^{(1)}}$ and $L^{\prime(1)}=L^{(1)}$. By the same argument as in the proof of Theorem 1.2, we see from this relation that $\Phi^{(1)}$ is a translation of $\mathbf{C}^{k}$, or $\Phi^{(1)}$ is given by $\Phi^{(1)}\left(w^{(1)}\right)=w^{(1)}+\beta^{(1)}$ for $w^{(1)} \in T_{\Xi^{(1)}}$. Since the base $\Xi^{(1)}$ of $T_{\Xi^{(1)}}$ is a convex domain in $\mathbf{R}^{k}$ containing no complete straight lines, it follows that $\beta^{(1)} \in \sqrt{-1} \mathbf{R}^{k}$. We observe that $\Phi^{(2)}$ satisfies

$$
\Phi^{(2)}(w+\sqrt{-1} L m)=\Phi^{(2)}(w)+\sqrt{-1} m^{(2)} \text { for every } w \in T_{\Xi} \text { and every } m \in \mathbf{Z}^{n},
$$

where we write $m^{(2)}={ }^{t}\left(m_{k+1}, \ldots, m_{n}\right)$ for $m={ }^{t}\left(m_{1}, \ldots, m_{n}\right)$.

As a consequence of our observations above, we know how to construct an element of $\operatorname{Aut}(D)^{\circ}$ from an element of $\operatorname{Aut}\left(\left(\mathbf{C}^{*}\right)^{l}\right)^{\circ}$. To see this, let $\theta$ be any element of $\operatorname{Aut}\left(\left(\mathbf{C}^{*}\right)^{l}\right)^{\circ}$ and take an element $\tilde{\theta}$ of $\operatorname{Aut}\left(\mathbf{C}^{l}\right)$ given as a lifting of $\theta$. We define an automorphism $\Phi$ of $T_{\Xi}$ by putting $\Phi^{(1)}\left(w^{(1)}\right)=w^{(1)}$ and $\Phi^{(2)}(w)=\tilde{\theta}\left(w^{(2)}\right)$ in (3.15) with $T_{\Xi^{\prime}}=T_{\Xi}$ and $T_{\Xi^{\prime}(1)}=T_{\Xi^{(1)}}$. Then, noting that

$$
\begin{aligned}
\Phi^{(2)}(w+\sqrt{-1} L m) & =\tilde{\theta}\left(w^{(2)}+\sqrt{-1} m^{(2)}\right) \\
& =\tilde{\theta}\left(w^{(2)}\right)+\sqrt{-1} m^{(2)}=\Phi^{(2)}(w)+\sqrt{-1} m^{(2)}
\end{aligned}
$$

for every $w \in T_{\Xi}$ and every $m \in \mathbf{Z}^{n}$, 
we see that $\Phi$ satisfies (3.14) with $T_{\Xi^{\prime}}=T_{\Xi}$ and $L^{\prime}=L$. Therefore the automorphism $F_{L}{ }^{-1} \circ \Phi \circ F_{L}$ of $T_{\Omega}$ satisfies

$$
\begin{aligned}
F_{L}{ }^{-1} \circ \Phi \circ F_{L}(\zeta+\sqrt{-1} m)= & F_{L}{ }^{-1} \circ \Phi \circ F_{L}(\zeta)+\sqrt{-1} m \\
& \text { for every } \zeta \in T_{\Omega} \text { and every } m \in \mathbf{Z}^{n},
\end{aligned}
$$

and induces an automorphism $\varphi$ of $D$ with $A(\varphi)=I_{n}$.

\section{REFERENCES}

[ 1 ] D. BARRETT, E. BEDFORD AND J. DADOK, $T^{n}$-actions on holomorphically separable complex manifolds, Math. Z. 202 (1989), 65-82.

[2] M. JARnicKi AND P. Pflug, First steps in several complex variables: Reinhardt domains, EMS Textbooks in Mathematics, European Mathematical Society, Zürich, 2008.

[3] S. ShImizU, Automorphisms and equivalence of bounded Reinhardt domains not containing the origin, Tohoku Math. J. 40 (1988), 119-152.

[ 4 ] S. ShimizU, Automorphisms of bounded Reinhardt domains, Japan. J. Math. 15 (1989), 385-414.

[ 5 ] S. ShimizU, Holomorphic equivalence problem for a certain class of unbounded Reinhardt domains in $\mathbf{C}^{2}$, Osaka J. Math. 28 (1991), 609-621.

[ 6 ] S. Shimizu, Holomorphic equivalence problem for a certain class of unbounded Reinhardt domains in $\mathbf{C}^{2}$, II, Kodai Math. J. 15 (1992), 430-444.

[7] S. ShimizU, A classification of two-dimensional Reinhardt domains, in preparation.

Mathematical Institute

TOHOKU UNIVERSITY

SENDAI 980-8578

JAPAN

E-mail address: shimizu@ math.tohoku.ac.jp 